\title{
JSH Statement: Tokyo declaration promoting salt reduction by the Japanese Society of Hypertension-the JSH Tokyo declaration
}

\author{
Takuya Tsuchihashi $^{1} \cdot$ Toshihiko Ishimitsu $^{2} \cdot$ Katsuyuki Ando $^{3} \cdot$ Miho Kusaka $^{4}$ Atsuhiro Ichihara ${ }^{5}$. \\ Katsuyuki Miura $^{6} \cdot$ Yuhei Kawano $^{7} \cdot$ Takafumi Ishida $^{8} \cdot$ Hiroo Kumagai $^{9}$ - Atsushi Sakima ${ }^{10} \cdot$ Hiroyuki Takase $^{11}$. \\ Hitomi Hayabuchi ${ }^{12} \cdot$ Hiroshi Itoh $^{13}$
}

Received: 28 April 2020 / Accepted: 29 April 2020 / Published online: 1 June 2020

(c) The Japanese Society of Hypertension 2020

The number of people with hypertension in Japan is estimated to be 43 million. The guidelines for the management of hypertension by the Japanese Society of Hypertension 2019 (JSH2019) defined blood pressure (BP) levels of 130-139/80-89 mmHg as 'elevated blood pressure' [1]. The JSH2019 also revised the target level of BP control to the lower level. Thus, lifestyle modifications are quite important in addressing hypertension regardless of the use of antihypertensive drugs. Among lifestyle modifications, salt reduction is most important, especially in Japanese individuals who consume significant amounts of salt. Based on the National Health and Nutrition Survey of Japan in 2018 , daily salt intake is reported to be $11.0 \mathrm{~g}$ for men and $9.3 \mathrm{~g}$ for women [2]. Thus, great effort is necessary to achieve the target salt intake level of $<6 \mathrm{~g} /$ day recommended for hypertensive patients in the JSH2019.

The JSH announced the "Tokyo Declaration Promoting Salt Reduction by the Japanese Society of Hypertension-the JSH Tokyo declaration" at the 42nd Annual Scientific
Meeting in Tokyo. We pledge to undertake a specific action plan comprising the following six strategies to achieve the target salt intake level of $<6 \mathrm{~g} /$ day.

The JSH will:

(1) Educate citizens on the harms of excessive salt intake and the importance of reducing salt intake.

(2) Recommend the assessment of the salt intake of individuals or populations and propose adequate methods to reduce salt intake.

(3) Promote salt reduction for children as a part of dietary education at school.

(4) Promote salt reduction in takeout food, prepared dishes, canteens, and school lunches.

(5) Encourage companies to develop and promote lowsalt foods.

(6) Encourage the government to take measures to promote salt reduction.
Takuya Tsuchihashi

tuti@ns.yawata-mhp.or.jp

1 Department of cardiology and Hypertension, Steel Memorial Yawata Hospital, Kitakyushu, Japan

2 Department of Nephrology and Hypertension, Dokkyo Medical University, Mibu, Japan

3 Iguchi Nephrology-Urology-Internal Medicine Clinic Shin-Koiwa, Tokyo, Japan

4 Kusaka Clinic, Kure, Japan

5 Department of Endocrinilogy and Hypertension, Tokyo Women's Medical University, Tokyo, Japan

6 Department of Public Health, Shiga University of Medical Science, Otsu, Japan
7 Department of Medical Technology, Teikyo University Fukuoka, Omuta, Japan

8 Department of Cardiovascular Medicine, Fukushima Medical University, Fukushima, Japan

9 Department of Nephrology and Endocrinology, National Defense Medical College, Tokorozawa, Japan

10 Health Administration Center, University of the Ryukyus, Okinawa, Japan

11 Department of Internal Medicine, JA Shizuoka Kohseiren Enshu Hospital, Hamamatsu, Japan

12 Department of Food Science and Nutrition, Faculty of Human Life and Environment, Nara Women's University, Nara, Japan

13 Department of Endocrinology, Metabolism and Nephrology, School of Medicine, Keio University, Tokyo, Japan 


\section{Compliance with ethical standards}

Conflict of interest The authors declare that they have no conflicts of interest.

Publisher's note Springer Nature remains neutral with regard to jurisdictional claims in published maps and institutional affiliations.

\section{References}

1. Umemura S, Arima H, Arima S, Asayama K, Dohi Y, Hirooka Y, et al. The Japanese Society of Hypertension. Guidelines for the Management of Hypertension (JSH2019). Hypertens Res. 2019;42:1235-481.

2. The Ministry of Health, Labour, and Welfare. The results of the 2018 National Health and Nutrition Survey in Japan. 2020. https://www.mhlw.go.jp/content/10900000/000615383.pdf. 